Article

\title{
Cognitive Network Science Reconstructs How Experts, News Outlets and Social Media Perceived the COVID-19 Pandemic
}

\author{
Massimo Stella ${ }^{1,2}$ (D) \\ 1 Department of Computer Science, University of Exeter, Exeter EX4 4QF, UK; massimo.stella@inbox.com \\ 2 Complex Science Consulting, Via Amilcare Foscarini 2, 73100 Lecce, Italy
}

Received: 27 August 2020; Accepted: 26 October 2020; Published: 29 October 2020

check for updates

\begin{abstract}
This work uses cognitive network science to reconstruct how experts, influential news outlets and social media perceived and reported the news "COVID-19 is a pandemic". In an exploratory corpus of 1 public speech, 10 influential news media articles on the same news and 37,500 trending tweets, the same pandemic declaration elicited a wide spectrum of perceptions retrieved by automatic language processing. While the WHO adopted a narrative strategy of mitigating the pandemic by raising public concern, some news media promoted fear for economic repercussions, while others channelled trust in contagion containment through semantic associations with science. In Italy, the first country to adopt a nationwide lockdown, social discourse perceived the pandemic with anger and fear, emotions of grief elaboration, but also with trust, a useful mechanism for coping with threats. Whereas news mostly elicited individual emotions, social media promoted much richer perceptions, where negative and positive emotional states coexisted, and where trust mainly originated from politics-related jargon rather than from science. This indicates that social media linked the pandemics to institutions and their intervention policies. Since both trust and fear strongly influence people's risk-averse behaviour and mental/physical wellbeing, identifying evidence for these emotions is key under a global health crisis. Cognitive network science opens the way to unveiling the emotional framings of massively read news in automatic ways, with relevance for better understanding how information was framed and perceived by large audiences.
\end{abstract}

Keywords: COVID-19; computational cognitive science; semantic networks; text mining; social media mining; emotions

\section{Introduction}

On 11 March 2020 the World Health Organization (WHO) declared COVID-19 a pandemic. Simultaneously, a secondary infodemic of COVID-19 news flooded information systems, overwhelming large audiences with a deluge of content about the COVID-19 spread [1]. In addition to this infodemic of fake news, even fact-checked articles reported a wide variety of contrasting views about the pandemic, e.g., diminishing the threat posed by the pathogen, creating alarm about the impact of the novel coronavirus over the economy or trying to convey the importance of social distancing for containing virus spreading. This massive proliferation of multiple views about the novel coronavirus overwhelmed large audiences with limited attention, ultimately promoting anxiety and inhibiting people from understanding the exact and correct dynamics of the pandemic [2].

In order to reduce anxiety and stress, identifying the contents promoted by this "battle of ideas" is urgently needed, also given how semantic and emotional content can deeply influence how we perceive and respond to massive events [2-4].

This manuscript outlines how recent tools from computational cognitive science [5,6] can crucially reconstruct how experts, news media and social media perceived and discussed the COVID-19 
pandemic. Theoretical grounding is provided by semantic frame theory, which indicates that the meaning attributed in language to individual words or concepts is fundamentally built over conceptual associations with other concepts [7-9]. Hence, meaning cannot be attributed to words/concepts in isolation but it rather requires access to their semantic frame, i.e., the set of concepts linked to a given conceptual entity as occurring in language. Therefore, understanding the cognitive reflection attributed by individuals to the real world in terms of perceived meaning depends on the semantic frame in which concepts are entwined together. For instance, in a text if "pandemic" is conceptually related to other jargon like "disease", "spreading" and "pathogen" then the text author(s) framed "pandemics" as a phenomenon related to disease diffusion. Conceptual relationships might be of different types, like word co-occurrences (see also the interesting historical investigation of "risk" in [10]) or syntactic/semantic links (like words specifying each other in a sentence or possessing the same meaning, see also [5]). Semantic frames arise from the associative structure of language and contain both semantic and emotional content $[9,11]$. Recent research pointed out how different populations can reconstruct semantic frames containing very different emotions around the very same concept, e.g., online users related the very same hashtag \#coronavirus to more pronounced fear-eliciting content when discussing the medical emergency and to more trustful concepts when discussing lockdown and other measures preventing contagion [12]. The identification of the emotional content of a given portion of language is called emotional profiling and it has been recently applied also to the investigation of online perceptions of COVID-19 [12-15].

This manuscript combines semantic frame theory with emotional profiling in order to reconstruct the plurality of views and emotions on the COVID-19 pandemic that were presented to millions of individuals by: (i) the authoritative WHO declaration of 11 March 2020, (ii) influential news media reporting such declaration and (iii) online social media. Attention is given to the first news that officially declared COVID-19 as a global pandemic for the whole world and not limited to Wuhan or China only. This news, "COVID-19 is a pandemic", started with the declaration by the WHO dated 11 March 2020 and it then reverberated across the above information channels. The structure of the paper includes a Methods section, outlining the linguistic datasets and the network analysis used here, followed by a Results section, reporting on the semantic frames and emotional profiles across eight basic emotions [11] of all the above declarations/articles/social media discourse. A Discussion section comments on the plurality of semantic views found in this exploration. The Discussion also interprets such results in light of relevant theories from cognitive science and other studies about the psychological impact of COVID-19 in relation to anxiety management, grief elaboration and psychological distress.

\section{Material and Methods}

This exploratory study focused on how COVID-19 was perceived by different information channels and investigated a corpus including three different sources of textual data: 1 speech transcript (WHO declaration), 10 news media articles on the same news (i.e., reporting the WHO declaration) and 37,500 tweets from social media (discussing the COVID-19 pandemic in the immediate aftermath of the WHO declaration). Notice that the requirement for articles to be on the same news crucially reduced the sample size of investigated texts.

This section outlines relevant information about the datasets and methods used in the main text.

\subsection{Data Access: SPEECH Transcript and News Media Articles}

The main analysis investigated the structure of linguistic knowledge as expressed in texts. This study did not generate any new data. The 10 news media analysed here were identified as influential by using a Google query as implemented in Mathematica 11.3. The Google query "COVID-19 WHO pandemic" on 1 April 2020 provided the top-ranked ten articles reported in Table 1, mostly produced between 10 and 12 March 2020. Notice that all investigated articles were: 
(1) Produced, published and updated between 10 March and 22 March, which is the immediate aftermath of the WHO declaration of the COVID-19 outbreak being a pandemic (dated 10 March 2020). A total of 9 out of 10 articles were produced and updated between 10 March and 12 March, guaranteeing a temporal coherence of the considered dataset, i.e., a collective of articles being produced within the same time window of the investigated Twitter dataset (see also Section 2.2);

(2) As reported in their titles, all the investigated articles focused on the same news of COVID-19 being declared a pandemic by the WHO. This is an important indicator that the considered news articles did not focus on local happenings but rather analysed and interpreted the COVID-19 pandemic in light of the WHO declaration.

Table 1. News media articles investigated in this study, with online links and labels (Media 1-10).

\begin{tabular}{|c|c|c|c|}
\hline Media ID and Link & News Outlet & Article Title & Number of Words \\
\hline Media1 & ABC News & $\begin{array}{l}\text { What the WHO pandemic } \\
\text { declaration means. }\end{array}$ & 748 \\
\hline Media2 & Business Insider & $\begin{array}{l}\text { The coronavirus is officially a } \\
\text { pandemic. }\end{array}$ & 576 \\
\hline Media3 & $\mathrm{BBC}$ & $\begin{array}{l}\text { Coronavirus: What is a pandemic } \\
\text { and why use the term now? }\end{array}$ & 318 \\
\hline Media4 & Channel News Asia & $\begin{array}{l}\text { Threat of coronavirus pandemic } \\
\text { now "very real": WHO }\end{array}$ & 553 \\
\hline Media5 & New Scientist & $\begin{array}{l}\text { COVID-19: Why won't the WHO } \\
\text { officially declare a coronavirus } \\
\text { pandemic? (Updated } 11 \text { March) }\end{array}$ & 801 \\
\hline Media6 & National Geographic & $\begin{array}{c}\text { Coronavirus is officially a } \\
\text { pandemic. }\end{array}$ & 1588 \\
\hline Media7 & $\mathrm{CNBC}$ & $\begin{array}{c}\text { World Health Organization } \\
\text { declares the coronavirus outbreak } \\
\text { a global pandemic }\end{array}$ & 939 \\
\hline Media8 & Telegraph & $\begin{array}{l}\text { Coronavirus outbreak declared a } \\
\text { pandemic: what does it mean, and } \\
\text { does it change anything? }\end{array}$ & 1387 \\
\hline Media9 & Times & $\begin{array}{l}\text { World Health Organization } \\
\text { Declares COVID-19 a "Pandemic." }\end{array}$ & 625 \\
\hline Media10 & Washington Post & $\begin{array}{l}\text { WHO declares a pandemic of } \\
\text { coronavirus disease COVID-19 }\end{array}$ & 1035 \\
\hline
\end{tabular}

The speech transcript of the WHO declaration of COVID-19 being a pandemic was obtained from the official website of the WHO (www.who.int, Last Accessed: 22 September 2020) and it included 778 words.

\subsection{Data Access: Social Media Tweets}

This analysis featured 37,500 Italian trending tweets gathered by Complex Science Consulting through the Twitter-approved account @ConsultComplex between 11 March and 17 March 2020. All the investigated tweets had to feature the hashtag \#pandemic together with one of the following hashtags: \#coronavirus, \#COVID-19, \#COVID. For instance, tweets featuring both \#pandemic and \#COVID were included in the analysis. Only tweets identified as trending by the Mathematica 11.3 "Popular" flag in the Twitter crawler ServiceConnect [] were considered in this analysis. The analysis focused on Italy given its resonance across the media due to the dramatic escalation of COVID-19 contagions in March 2020. A different dataset was also crawled by Complex Science Consulting within the same time period and investigated separately by [12]. 
Media links and pictures were discarded. Whereas in other studies, like [12], hashtags were used in order to infer different topics embedded in tweets, in which hashtags were integrated with other words in text. Hashtags were translated in Italian words, where possible, by using a simple overlap rule between the hashtag content with no \\# symbol and Italian words (e.g., \#COVID became "COVID"). Tweets in languages different from Italian were not considered in this analysis. The resulting dataset included 37,500 tweets, each one treated as a string of text. No user-level information was used in the investigation (e.g., usernames, number of followers, etc.). No tweet-level information was used (e.g., number or likes, number of retweets, etc.), except for the timestamp, which was used in order to select only messages produced between 11 March and 17 March 2020.

Although this work focused on Italian social media, the investigated tweets were influenced by the WHO declaration too and they were gathered in the next few days after the declaration itself. Hence, the social discourse analysed here cannot be considered independent from the views portrayed in news articles. Establishing a direct causal link between exposure to news articles and social media reactions goes beyond the scope of this explorative study and it represents a delicate research question that started being successfully explored only in recent studies [16,17]. Instead, this investigation focuses on comparing the semantic frames and emotional perceptions about the COVID-19 pandemic in the aftermath of the WHO declaration across different online sources.

\subsection{Forma Mentis Networks as Knowledge Graphs Extracted from Text}

This manuscript adopted the recent framework of forma mentis networks-as already introduced in previous studies-that used automatic text processing for reconstructing how social media discussed the gender gap in science [5] and for exploring online perceptions and emotions in Italy after the release of national lockdown [13]. On the one hand, [5] also showed that textual forma mentis networks in annotated texts are successful in determining the topic of a text. On the other hand, [13] showed that textual forma mentis networks are sensitive enough to highlight flickering emotions over time in the social discourse around specific topics and hashtags. The interested reader is referred to these prior works for more details. Here, the methodology of textual forma mentis networks is reported in a concise yet self-contained manner. Based on cognitive data and text processing, forma mentis networks are knowledge graphs [5] representing the mental lexicon, a cognitive system storing linguistic information and driving word acquisition and use [6,9]. Representing conceptual associations between words as a network of nodes (words) and links (syntactic/semantic associations), textual forma mentis networks (TFMNs) extract both knowledge and affective patterns, as embedded in the text and are representative of a given author's mindset. Other approaches also represented texts as complex networks though they focused mainly on co-occurrences between words, did not consider the cognitive/emotional dimensions of words but were successful in identifying patterns like writing styles [18]. Several successful approaches also used syntactic relationships in order to investigate how words tend be assembled in sentences in order to minimise dependency crossings and thus, potential confusions about understanding knowledge and its meaning (for a review, see [19]). Here, TFMNs focus on associative knowledge, linking together concepts according to meaning specifications provided in language (see [5]). When we read sentences, we do not explicitly observe such a conceptual network, but we mentally reconstruct it in order to understand meaning. Consider the example "pandemics and diseases are terrible", a sentence specifying that both the concepts "pandemic" and "disease" have a specific feature, i.e., being "terrible". A textual forma mentis network would represent such a sentence as a network connecting the conceptual nodes "pandemic" and "disease" to "terrible". In other words, textual forma mentis networks highlight the syntactic network of conceptual associations underlying a given text and conveying a given meaning. TFMNs thus reconstruct the syntactic and semantic structure of texts, also providing the advantage of visualising, highlighting and exploring conceptual associations in texts under the lens of network science. Syntactic relationships were extracted by using the language models available in TextStructure[], a command implemented in Mathematica 11.3. For instance, in the sentence "hospitals are full" there is a syntactic relationship specifying 
a property (being "full") of "hospitals", thus linking "hospital" (stem for "hospitals") and "full". Semantic relationships indicating meaning overlap (i.e., "disease" and "sickness" being synonyms in some contexts) were retrieved by using WordNet 3.0 in English and its Italian translation [20]. All words in texts were stemmed in order to represent different inflected forms with the same concept (e.g., "talking", "talked" and "talks" all representing "talk"). English words were stemmed by using the WordStem[] function implemented in Mathematica 11.3. Stemming considers only the core of a word, discarding inflections and suffixes (e.g., "learning" and "learns" would be represented with the same stem "learn"). In Italian, words were stemmed by using the Snowball C stemming algorithm as implemented in R. Italian words were translated into English for easier visualisation.

Figure 1 provides a visual scheme of the different operations leading from textual data to reconstructed, quantitative perceptions of a given topic or keyword. It must be underlined that the application of the same unsupervised methodology across different texts enables a quantitative comparison of the semantic content and emotional portrayals included in the language used by different authors. The cognitive datasets enabling emotional profiling are described in the next Section.

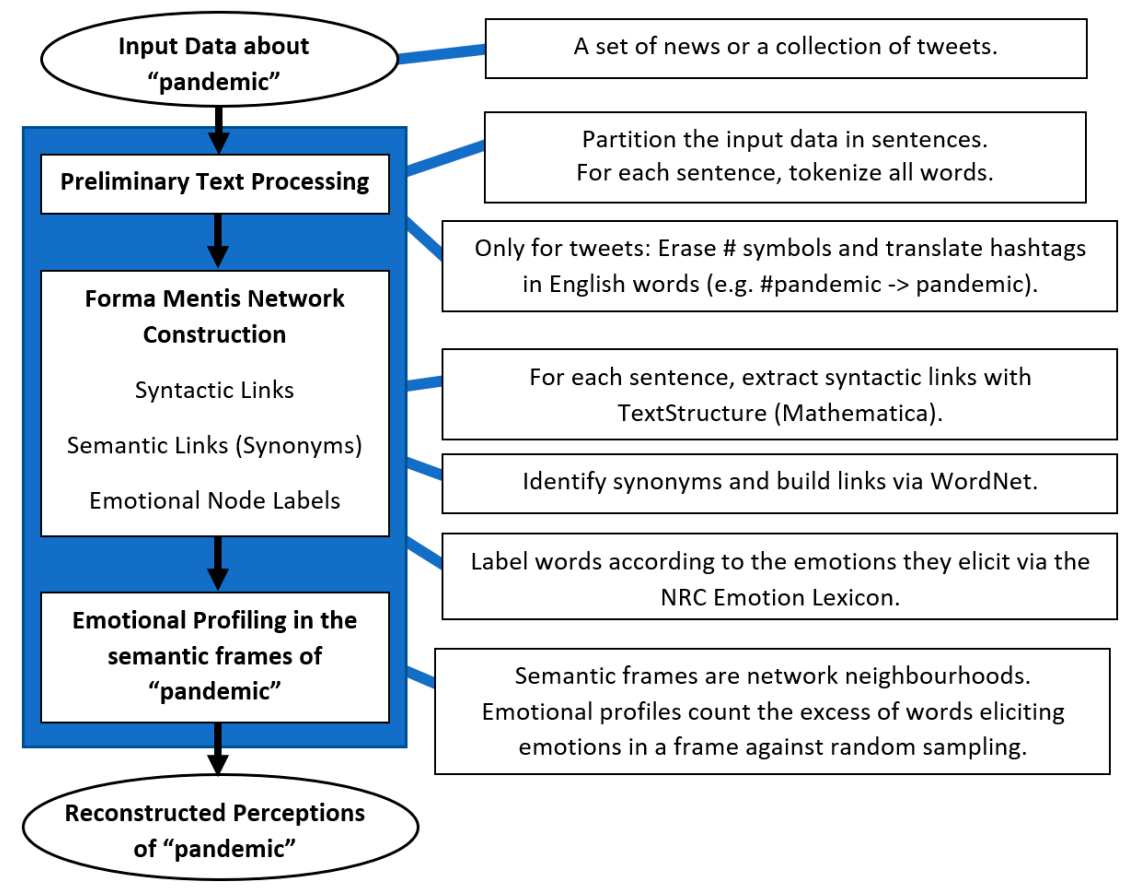

Figure 1. A scheme of the different phases leading to reconstructing emotional profiles and semantic content in texts via textual forma mentis networks.

\subsection{Emotional Profiling and Cognitive Datasets}

Combined with the Emotion Lexicon by Mohammad and Turney [21], TFMNs can identify the emotional profile of a given concept in a collection of texts. The emotional profile of a concept $C$, here was defined as the number of words eliciting a given emotion associated/linked to $C$, i.e., words in the network neighbourhood of $C[5,12,13]$. Hence, the emotional profile of a concept in a forma mentis network was computed by counting how many words elicited a given emotion in the network neighbourhood of a concept (e.g., 35\% of the neighbours of "pandemic" elicited fear). The emotions considered here were the ones included in the NRC Emotion Lexicon, namely: anger, anticipation, disgust, fear, joy, sadness, surprise and trust. In counting the associations giving rise to a given emotional profile, negations were considered by associating antonyms to negated words. In presence of words exclusively linked to a negation (e.g., "love" and "not"), antonyms directly substituted negated words (e.g., "hate" was considered for the emotional profiling, replacing "love" and "not"). In the presence of words linked to a negation and to other concepts of the same sentiment (e.g., "love" 
connected to both "not" and "faith"), both antonyms and negated words were considered (e.g., "hate" and "love" were both considered for the emotional profiling, replacing "love" and "not"). The NRC Emotion Lexicon in Italian was obtained from the English one by adopting a consensus translation with services including Google Translate, Bing Translator and DeepL. Where at least two out of three translator services provided the same translation for a word, that one was used. In all other cases translation was human coded by the author. On a random sample of 200 words, the accuracy of translation was estimated to be $93 \%$. This required also an additional human coding of the translation, checking for and correcting mistakes according to the author's expertise.

Counting words provides an emotional profile indicating how rich a given semantic frame/network neighbourhood is in terms of words eliciting the above emotions. However, the NRC lexicon features more words eliciting certain emotions and fewer words eliciting other emotional states, so that considering word counting by itself might be provide biased results if no null model is considered. In the current study, the null model for emotional profiles was random word sampling from the NRC lexicon, i.e., sampling at random from the lexicon as many words as observed in the emotional profile and computing the relative random emotional profile. Z-scores were computed between the empirical emotional profiles of "pandemic" and a distribution of 1000 random samples. The confidence interval relative to a 0.05 significance level was adopted as the neutrality range, i.e., a range where no emotional pattern was identified.

Notice that emotional profiles were visualised through emotion wheels, following the visualisation method originally proposed by Plutchik [11]. A blue/grey/red colour scheme was used in order to cluster together negative emotions (in red), neutral emotions (in grey) and positive emotions (in blue).

\section{Results}

Semantic frames and emotions, as attributed in the processed texts, highlight interesting patterns on how the COVID-19 pandemic was announced by WHO and discussed by news outlets and social media users.

\subsection{Investigating the Knowledge and Emotions in the Whole WHO Declaration}

Figure 2 visualises the WHO declaration of "COVID-19 is a pandemic" in terms of a knowledge graph, a textual forma mentis network representing the mindset (in Latin forma mentis) portrayed in the declaration in terms of concepts (nodes) linked by semantic and syntactic relationships (cf. Methods). Larger nodes are relative to concepts with a higher closeness centrality (see [22]), i.e., connected by fewer links to all other concepts in the network. Since previous studies indicated how closeness in forma mentis networks captures semantic prominence (see [5]), the knowledge graph indicates how the WHO declaration mainly revolved around "country". Other prominent concepts (i.e., high in closeness centrality) were "we", "not", "take", "case", "change", "tell" and "numbers", indicating that the speech adopted a first-person narrative revolving around countries and the change (of the pandemic) due to the registered numbers of cases. This is not surprising but comes as a confirmation that a network metric (closeness centrality) can identify key aspects of an apparently unstructured piece of text (in this case the WHO declaration of COVID-19 being a pandemic).

In addition to this reality check, the knowledge graph also visually represents key aspects of a text. For instance, in Figure 2 words are clustered in communities as identified by a Louvain algorithm [22]. Communities are defined as network regions made of tightly interconnected nodes and in a forma mentis network a community can provide contextual knowledge describing the meaning attributed to concepts across sentences [5]. By considering the community including "pandemic" one can notice that the WHO speech presented the concept of a pandemic to the general audience in relationship to "health", "change", "crisis" and even "economy": the WHO presented the pandemic as a challenging crisis not only for global health but also for the economy. Positive terms like "protect", "together" and "calm" appeared within the same community of "pandemic" and indicate a tendency for the speech to call for calmness and a communal protection against such crisis. 


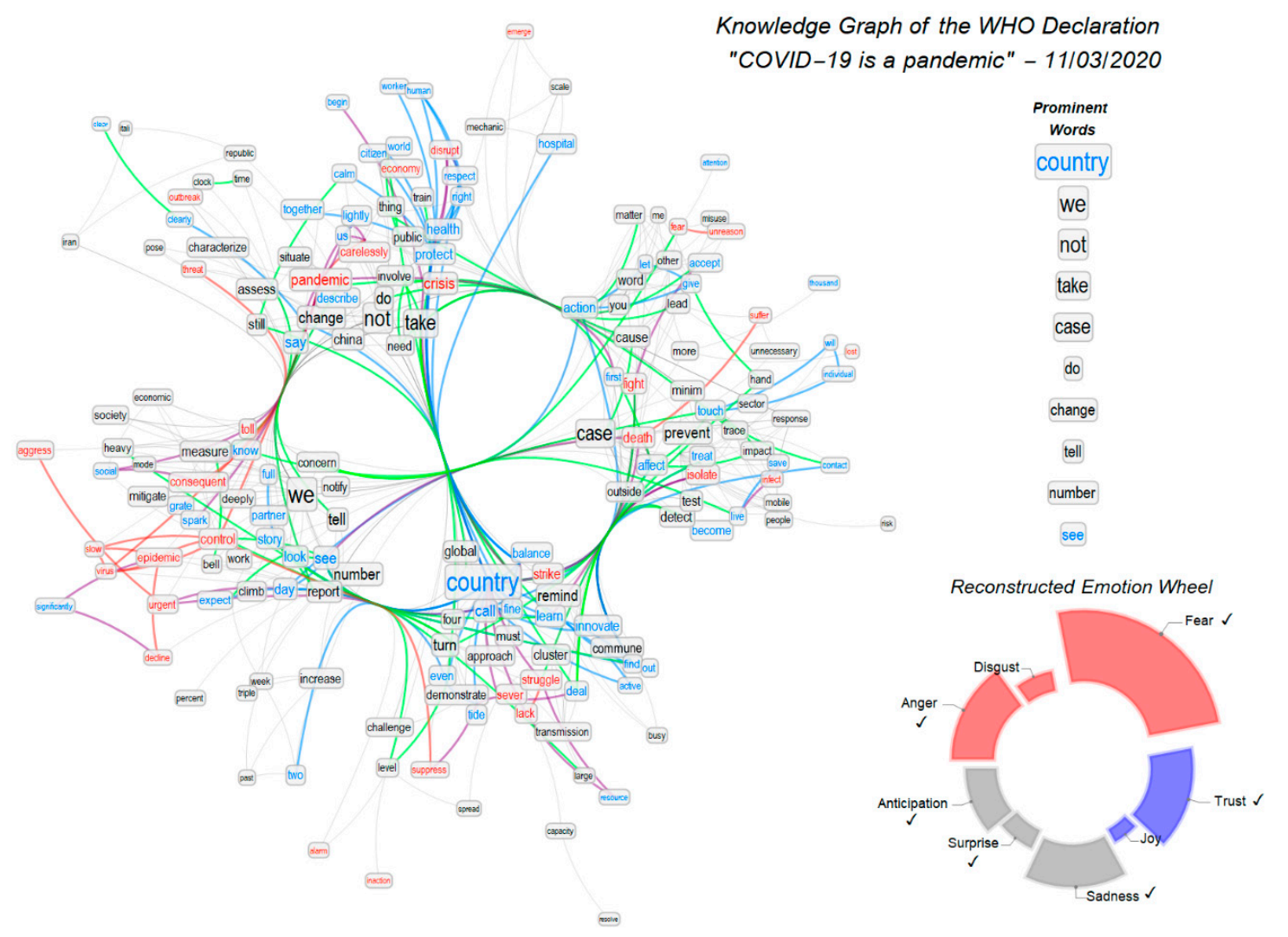

Figure 2. Left: Knowledge graph representing syntactic associations between words of positive (cyan), negative (red) and neutral (black) sentiment and semantic overlap between synsets (green) as extracted from the WHO Declaration of COVID-19 being a pandemic, 11 March 2020. Larger font size indicates higher closeness centrality and conceptual prominence in the text (see Section 2). Top right: Prominent words in the discourse as detected from syntactic and semantic associations. Bottom right: Emotion wheel, counting how many words elicited a given emotion in the knowledge graph. Emotions stronger than random expectation were marked with a check (Z-test against random word sampling, significance level of 0.05).

The overall emotional profile of the speech is reported in Figure 2 (bottom, right). The words adopted in the WHO declaration elicited strong emotional patterns of fear, anger and sadness but also levels of trust higher than random expectation. Combinations of fear and sadness indicate concern, according to Plutchik's wheel of emotions [11], but what about the combination of positive and negative emotions? The co-existence of concern and trust indicates a clear narrative strategy pursued by the WHO in their speech: to clearly explain the dangers posed by the COVID-19 emergency to the general public, while subsequently adopting also trustful language focusing on how to face the crisis itself.

\subsection{Investigating Knowledge and Emotions around "Pandemic" across Texts}

Forma mentis networks were built not only for the WHO declaration, but for all the considered articles and aggregated tweets. Rather than investigating the whole networks, attention was given to the individual semantic frames, i.e., the network neighbourhoods, of "pandemic" across texts. Figure 3 (top) reports the semantic frames and emotional profiles of "pandemic" in the WHO declaration, within two of the 10 investigated articles and within social discourse. Figure 3 (bottom) provides the complete spectra of emotional profiles across all the investigated media. 
(A) WHO Pandemic Declaration

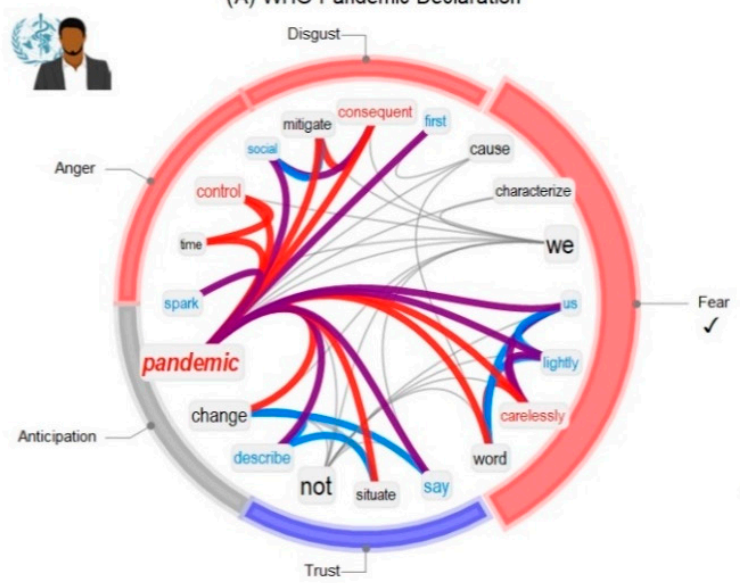

(C) 37,500 Italian Tweets

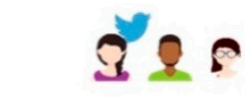

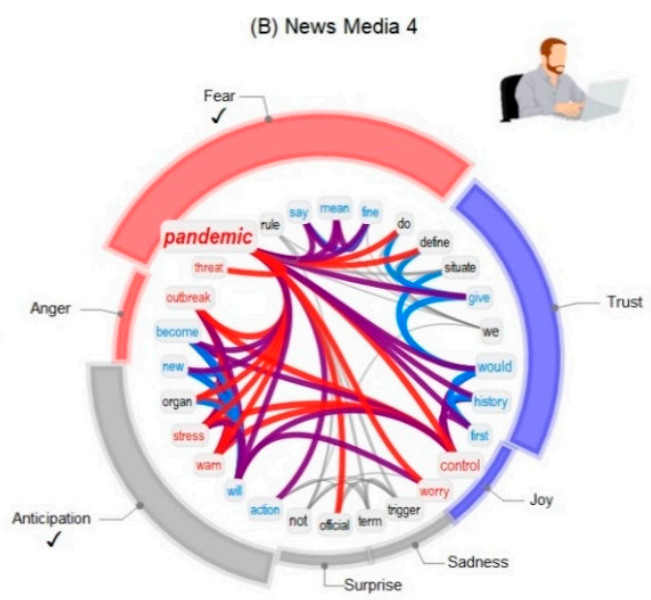

(D) News Media 7
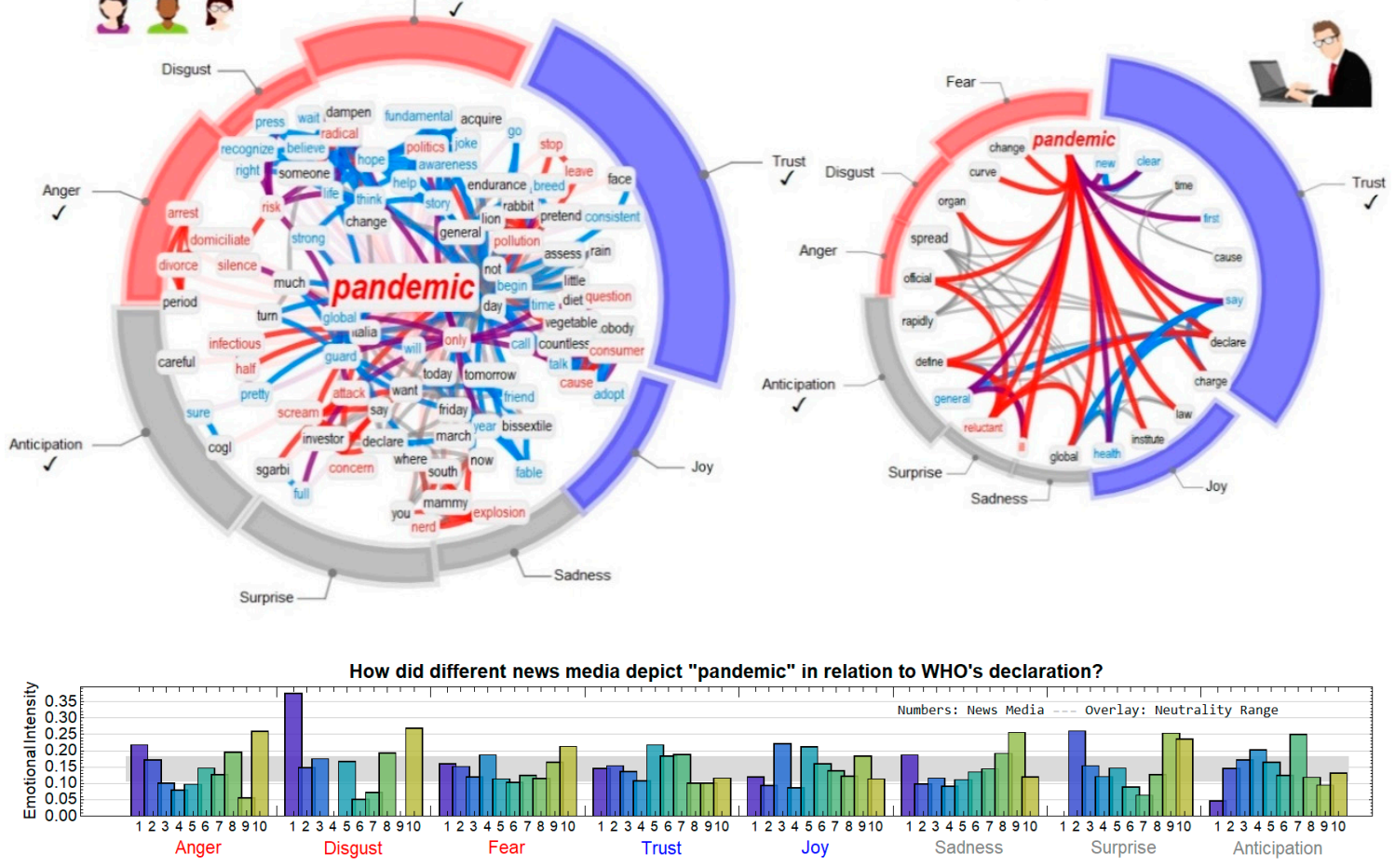

Figure 3. Top: Syntactic associations between positive (cyan), negative (red) and neutral (black) words as used in the WHO pandemic declaration (A), two influential news media reporting the WHO's declaration (B,D) and 37,500 tweets with \#pandemic posted right after the WHO's declaration (C). Emotions stronger than random expectation were marked with a check (z-test against random word sampling, significance level of 0.05). Larger concepts were more prominent in the semantic structure of the used language. Bottom: Emotional intensities of 10 different news media are reported. The grey bar in the foreground represents the error bound of neutral intensities (see Section 2).

As reported in Figure 3, the WHO declaration pictured "pandemic" as a phenomenon linked to control and mitigation, with associated concepts eliciting a moderate level of fear and expressing concern about the COVID-19 pandemic. Hence, the overall concern found in the WHO's whole declaration is present also around the specific semantic framing attributed by the WHO to "pandemic", as expected from a speech centred around massive pathogen diffusion.

The other semantic frames and emotional profiles reported here are the most dissimilar from the WHO's one. News media four provided a highly polarised perception of "pandemic", combining fear-inducing links to "threat" with more trustful concepts about the future, a combination indicating 
submission, apprehension and passive acceptance of COVID-19 being a pandemic. Instead, news media seven depicted a more neutral and trustful frame for the COVID-19 pandemic, using neutral jargon, explaining the epidemiological definition of a pandemic and conveying emotions of trust into waking up, getting ready and taking action. These patterns were absent in the original WHO declaration and have thus to be considered as cognitive alterations attributed to the way individual journalists framed the news "COVID-19 is a pandemic". It should be stressed that these alterations emerge from a purely quantitative modelling of texts and do not require any human coding or reading of the underlying texts.

Social media strongly debated over the COVID-19 pandemic. Here, attention was given to Italy, the first European country struck by COVID-19 and reacting with a national lockdown (see [12]). In 37,500 Italian tweets (see Section 2), "pandemic" spawned a social discourse featuring contrasting, affect-polarising concepts like "help", and "hope" together with "risk" and "attack". The resulting emotional profile was strongly trustful, dominated by a positive awareness of the coronavirus and the need to offer assistance against it, but also mixed with patterns of fear (against the risks of the pandemics), anger (against a global threat) and anticipation (for a better future).

Anger and fear are both natural evolutionary mechanisms for coping with threats [11] and are also natural responses in elaborating grief about a negative event, as indicated by the Kübler-Ross model [23]. These emotions are therefore expected when perceiving a pandemic and were also strongly detected in other studies focusing on different datasets related to social discourse about the COVID-19 pandemic (see [15]). Interestingly, in here grief-related emotions attributed to "pandemic" were found to be dominated by more positive ones, expressing awareness and willingness to act upon the recently declared health emergency. In this way, the perception of the "pandemic" itself is quantitatively found to be way richer in positive/trustful concepts than the framing portrayed by the WHO.

\section{Discussion and Conclusions}

The same event can be described in text through different semantic frames, eliciting different emotions and perceptions. The real challenge in AI is quantifying and reconstructing these frames without human coding and in the absence of training data. This manuscript tested knowledge modelling via cognitive network science, as a quantitative way for automatically identifying key concepts and their emotional perceptions in texts, e.g., articles and social media messages. The semantic and emotional frames reconstructed here around the news "COVID-19 is a pandemic" open quantitative ways for tracking how COVID-19 was discussed by experts, news media and social networks.

Fear, anger and trust about the pandemic were detected in news outlets and social media reaching large audiences. It is crucially important to keep track of any massive release of information vehiculating these emotions, since the latter can strongly influence an individuals' perception, mental well-being and real-world behaviour [11,24-26]. As recent studies from computational social science pointed out [27], both negative and positive emotions can give rise to emotional contagion over social media, a phenomenon where emotions pass from user to user by the mere act of reading a post/message portraying a certain emotional profile and without any real-world experience giving grounding to the transmitted emotions. Hence, emotional contagion over social media can infect unaware audiences and potentially de-stabilise public opinion about the COVID-19 crisis [1]. A recent investigation on the reopening after lockdown found that online users on Twitter tended to retweet more those messages richer in fear-eliciting jargon [13], further indicating the possibility for fear-eliciting content to give rise to emotional contagions of fear over social media.

According to cognitive science, contagions of fear and anger would have different repercussions. Responding to threats with anger greatly inhibits risk perception [24], leading to resisting risk-reduction policies (e.g., lockdown or social distancing). Instead, fear heightens risk perception, thus leading to more risk-averse behaviour [24]. However, prolonged states of fear are detrimental for correctly identifying threats and can also induce higher levels of cortisol, which impair both mental wellbeing and the immune system [26]. Hence, a deluge of fear-inspiring semantic frames, like the ones found in some news media in this exploratory study, might have concrete repercussions over the COVID-19 
contagion, influencing people's immune systems and bolstering their susceptibility to pathogens. The exact quantification of this relationship between COVID-19 fear levels and health susceptibility remains an open question for future research. However, it should be noted that fear can also assume a positive role in hampering the pandemics. In a sample of 324 people, Harper and colleagues [28] recently found that moderate levels of fear were functional in promoting public health compliance and boosting positive behavioural changes (e.g., social distancing) aimed at containing the contagion. This positive role played by fear aligns with the moderate levels of fear found in the semantic frame of "pandemic" in the WHO declaration, providing further support to the narrative expedient adopted by the WHO of raising concern about the novel coronavirus, with concerning jargon related to epidemics and also including mitigation measures.

Trust was identified in the semantic frames of "pandemic" as discussed by social media and some news outlets. The reconstructed semantic frame reported here indicated how news outlets channelled trust through scientific knowledge, a "scientific explanation" strategy useful for improving individuals' awareness about the mechanisms driving the pandemic and its containment. Recent studies also outlined how eliciting trust is a good strategy not only for reducing the incidence of fear and anger but also in order to boost compliance with regulations and responsible health behaviour [29]. The "scientific explanation" strategy was not found in the semantic frames extracted from social media discourse, where trust-eliciting concepts were not linked to scientific jargon but were rather connected to "politics". This confirms that trust can indeed come from different sources, as also channelling feelings of trust towards politics can make large audiences comply with public health orders like respecting social distancing $[29,30]$. Notice that higher levels of trust towards science and politics, as outlined here with this study, were also found by previous investigations targeting populations under recent lockdown caused by the COVID-19 pandemic [12,30].

Tracking the levels of perceived trust around the COVID-19 pandemic and identifying the sources of such trust, be it scientific knowledge or politics, is a crucial challenge for better understanding compliance with regulations. In fact, audiences with little trust in the institutions can mine the credibility of governments, both fostering divisive politics and hampering nationwide adoption of strategies for pandemic containment [29].

It should be noted that trust and fear are not direct opposites. According to the Atlas of Emotions [11], trust and awareness can turn anger into a constructive motivation towards facing the threat or source of anger itself-in this case the COVID-19 pandemic. This interconnectedness suggests a highly nuanced perception of social media users towards facing the COVID-19 pandemic, as also found within other studies focusing on Twitter data [12,14] and other social media platforms [1,15].

The above points outline a complex landscape of real-world implications for the different emotional perceptions of the COVID-19 pandemic. The current semantic/emotional frames can be a valid compass for navigating such landscape, with some limitations. This exploratory study considers only textual information, thus neglecting other types of communication through visual or verbal cues. Nonetheless, the plurality of perceptions quantified here, in a statistically robust way, indicate how already processing only textual sources might provide richly complex semantic frames and emotional profiles about how the COVID-19 pandemic is perceived, discussed and reported to large audiences.

Another limitation of this study was neglecting how potential automated accounts might have biased social discourse. Recent investigations [3,31] found that social bots, i.e., user accounts piloted by automatic software, were capable of influencing human behaviour by fostering negative, conspiratorial and anxious perceptions in online platforms. Applying the same tools of cognitive network science explored and tested here to the investigation of the semantic frames produced by social bots represents an interesting future research direction.

Besides the above limitations, the main advantage of cognitive network science is its ability to capture linguistic associations, semantic frames and emotions in a transparent way, enabling a visualisation of conceptual associations in texts and without requiring training data for machine learning. 
Future research should build upon cognitive network science for a better understanding of social media, possibly in synergy with other promising and successful automatic approaches to knowledge modelling [32-37]. Access to large-scale corpora of news media articles would enable prompt identification of outlets promoting distorted mindsets ("COVID-19 is just a flu") or panic-inducing misinformation. Additional cross-validation through careful human coding should be pursued as well, with the aim of further characterising how the semantic information highlighted by textual forma mentis networks is understood and processed by individuals. This calls for future research endeavours beyond the scope of the current preliminary study but always within the growing area of cognitive data science.

A richer understanding of the context of promoting negative perceptions should also be pursued in the analysis of political debate, like in [33], where cognitive networks can unveil how political parties or politicians discuss the COVID-19 emergency. The current methodology can also identify how people perceive health risks and concrete symptoms of COVID-19, integrating recent powerful analyses extracting the symptoms of the novel coronavirus from social discourse [35] and complementing interesting dynamical patterns of grief elaboration and COVID-19 recently unearthed in social platforms by Aiello and colleagues [15]. Notice that the representation of textual knowledge in tweets produced here could also be integrated with other representations based on word embedding models $[32,34,36]$ and powering recent natural language approaches to identifying topics in tweets discussing COVID-19 [36,37]. Forma mentis networks and other models of natural language processing all aim towards the common direction of monitoring and understanding large volumes of messages with the ease of lightweight and automatic knowledge extraction methodologies.

In this time of uncertainty, about the evolution of the coronavirus pandemic and the impact of lockdowns, having a clear, misinformation-free perception of the COVID-19 pandemic is fundamental [38].The cognitive network science framework outlined in this work can tackle this challenge, effectively capturing and reproducing a plurality of views promoted about the COVID-19 pandemic. Access to such information opens new ways for policy makers to act based on how large audiences perceive the COVID-19 pandemic and its repercussions and thus represents an impactful direction for future research.

Funding: The author received no financial support for the research, authorship, and/or publication of this article.

Conflicts of Interest: The author declares no conflict of interest.

\section{References}

1. Cinelli, M.; Quattrociocchi, W.; Galeazzi, A.; Valensise, C.M.; Brugnoli, E.; Schmidt, A.L.; Zola, P.; Zollo, F.; Scala, A. The covid-19 social media infodemic. Sci. Rep. 2020, 10, 16598. [PubMed]

2. Garfin, D.R.; Silver, R.C.; Holman, E.A. The novel coronavirus (COVID-2019) outbreak: Amplification of public health consequences by media exposure. Health Psychol. 2020, 39, 355-357. [CrossRef] [PubMed]

3. Stella, M.; Ferrara, E.; De Domenico, M. Bots increase exposure to negative and inflammatory content in online social systems. Proc. Natl. Acad. Sci. USA 2018, 115, 12435-12440.

4. Gallotti, R.; Valle, F.; Castaldo, N.; Sacco, P.; De Domenico, M. Assessing the risks of "infodemics" in response to COVID-19 epidemics. arXiv 2020, arXiv:2004.03997.

5. Stella, M. Text-mining forma mentis networks reconstruct public perception of the STEM gender gap in social media. PeerJ Comput. Sci. 2020, 6, e295. [CrossRef]

6. Siew, C.S.; Wulff, D.U.; Beckage, N.M.; Kenett, Y.N. Cognitive Network Science: A Review of Research on Cognition through the Lens of Network Representations, Processes, and Dynamics. Complexity 2019, 2019, 2108423. [CrossRef]

7. De Deyne, S.; Kenett, Y.N.; Anaki, D.; Faust, M.; Navarro, D. Large-Scale Network Representations of Semantics in the Mental Lexicon. In Big Data in Cognitive Science; Routledge/Taylor \& Francis Group: Abingdon, UK, 2017; pp. 174-202.

8. Fillmore, C.J.; Baker, C.F. Frame semantics for text understanding. In Proceedings of the WordNet and Other Lexical Resources Workshop NAACL, Pittsburgh, PA, USA, 3-4 June 2001; Volume 6. 
9. Vitevitch, M.S. (Ed.) Network Science in Cognitive Psychology; Routledge: Abingdon, UK, 2019.

10. Li, Y.; Hills, T.; Hertwig, R. A brief history of risk. Cognition 2020, 203, 104344.

11. Plutchik, R. The nature of emotions. Am. Sci. 2001, 89, 344-350.

12. Stella, M.; Restocchi, V.; De Deyne, S. \#lockdown: Network-Enhanced Emotional Profiling in the Time of COVID-19. Big Data Cogn. Comput. 2020, 4, 14.

13. Stella, M. Social discourse and reopening after COVID-19: A post-lockdown analysis of flickering emotions and trending stances in Italy. PsyarXiv 2020. [CrossRef]

14. Lwin, M.O.; Lu, J.; Sheldenkar, A.; Schulz, P.J.; Shin, W.; Gupta, R.; Yang, Y. Global sentiments surrounding the COVID-19 pandemic on Twitter: Analysis of Twitter trends. JMIR Public Health Surveill. 2020, 6, e19447.

15. Aiello, L.M.; Quercia, D.; Zhou, K.; Constantinides, M.; Šćepanović, S.; Joglekar, S. How Epidemic Psychology Works on Social Media: Evolution of responses to the COVID-19 pandemic. arXiv 2020, arXiv:2007.13169.

16. Schmidt, A.L.; Peruzzi, A.; Scala, A.; Cinelli, M.; Pomerantsev, P.; Applebaum, A.; Gaston, S.; Fusi, N.; Peterson, Z.; Severgnini, G.; et al. Measuring social response to different journalistic techniques on Facebook. Humanit. Soc. Sci. Commun. 2020, 7, 17. [CrossRef]

17. Gozzi, N.; Tizzani, M.; Starnini, M.; Ciulla, F.; Paolotti, D.; Panisson, A.; Perra, N. Collective response to the media coverage of COVID-19 Pandemic on Reddit and Wikipedia. J. Med. Internet Res. 2020, 22, e21597.

18. Amancio, D.R. Probing the topological properties of complex networks modeling short written texts. PLoS ONE 2015, 10, e0118394. [CrossRef]

19. Baronchelli, A.; Ferrer-I-Cancho, R.; Pastor-Satorras, R.; Chater, N.; Christiansen, M.H. Networks in cognitive science. Trends Cogn. Sci. 2013, 17, 348-360. [CrossRef] [PubMed]

20. Bond, F.; Foster, R. Linking and extending an open multilingual wordnet. In Proceedings of the 51st Annual Meeting of the Association for Computational Linguistics, Sofia, Bulgaria, 4-9 August 2013; Volume 1, pp. 1352-1362.

21. Mohammad, S.M.; Turney, P.D. Emotions evoked by common words and phrases: Using mechanical turk to create an emotion lexicon. In Proceedings of the NAACL HLT 2010 Workshop on Computational Approaches to Analysis and Generation of Emotion in Text, Association for Computational Linguistics, Los Angeles, CA, USA, 5 June 2010; pp. 26-34.

22. Newman, M. Networks; Oxford University Press: Oxford, UK, 2018.

23. Kübler-Ross, E.; Kessler, D. On Grief and Grieving; Simon and Schuster: New York, USA, 2005.

24. Lerner, J.S.; Keltner, D. Fear, anger, and risk. J. Personal. Soc. Psychol. 2001, 81, 146. [CrossRef]

25. Moroń, M.; Biolik-Moroń, M. Trait emotional intelligence and emotional experiences during the COVID-19 pandemic outbreak in Poland: A daily diary study. Personal. Individ. Differ. 2020, 168, 110348.

26. Staufenbiel, S.M.; Penninx, B.W.; Spijker, A.T.; Elzinga, B.M.; van Rossum, E.F. Hair cortisol, stress exposure, and mental health in humans: A systematic review. Psychoneuroendocrinology 2013, 38, 1220-1235. [CrossRef]

27. Kramer, A.D.; Guillory, J.E.; Hancock, J.T. Experimental evidence of massive-scale emotional contagion through social networks. Proc. Natl. Acad. Sci. USA 2014, 111, 8788-8790. [CrossRef]

28. Harper, C.A.; Satchell, L.P.; Fido, D.; Latzman, R.D. Functional fear predicts public health compliance in the COVID-19 pandemic. Int. J. Ment. Health Addict. 2020, 1-14. [CrossRef]

29. Khemani, S. An Opportunity to Build Legitimacy and Trust in Public Institutions in the Time of Covid-19. World Bank Res. Policy Briefs 2020, 148256. [CrossRef]

30. Sibley, C.G.; Greaves, L.M.; Satherley, N.; Wilson, M.S.; Overall, N.C.; Lee, C.H.J.; Milojev, P.; Bulbulia, J.; Osborne, D.; Milfont, T.L.; et al. Effects of the COVID-19 pandemic and nationwide lockdown on trust, attitudes toward government, and well-being. Am. Psychol. 2020, 75, 618-630. [CrossRef] [PubMed]

31. Pozzana, I.; Ferrara, E. Measuring bot and human behavioral dynamics. Front. Phys. 2020, 8, 125. [CrossRef]

32. Lücking, A.; Brückner, S.; Abrami, G.; Uslu, T.; Mehler, A. Computational linguistic assessment of textbook and online learning media by means of threshold concepts in business education. arXiv 2020, arXiv:2008.02096.

33. Brito, A.C.M.; Silva, F.N.; Amancio, D.R. A complex network approach to political analysis: Application to the Brazilian Chamber of Deputies. PLoS ONE 2020, 15, e0229928. [CrossRef] [PubMed]

34. Choi, M.; Aiello, L.M.; Varga, K.Z.; Quercia, D. Ten Social Dimensions of Conversations and Relationships. In Proceedings of the Web Conference 2020, Taipei, Taiwan, 20-24 April 2020; pp. 1514-1525.

35. Murray, C.; Mitchell, L.; Tuke, J.; Mackay, M. Symptom extraction from the narratives of personal experiences with COVID-19 on Reddit. arXiv 2020, arXiv:2005.10454. 
36. Müller, M.; Salathé, M.; Kummervold, P.E. COVID-Twitter-BERT: A Natural Language Processing Model to Analyse COVID-19 Content on Twitter. arXiv 2020, arXiv:2005.07503.

37. Gencoglu, O. Large-scale, Language-agnostic Discourse Classification of Tweets During COVID-19. arXiv 2020, arXiv:2008.00461.

38. Larson, H.J. Blocking information on COVID-19 can fuel the spread of misinformation. Nature 2020, $580,306$.

Publisher's Note: MDPI stays neutral with regard to jurisdictional claims in published maps and institutional affiliations.

(C) 2020 by the author. Licensee MDPI, Basel, Switzerland. This article is an open access article distributed under the terms and conditions of the Creative Commons Attribution (CC BY) license (http://creativecommons.org/licenses/by/4.0/). 